NBER WORKING PAPER SERIES

\title{
CAN AUTOMATIC RETENTION IMPROVE HEALTH INSURANCE MARKET OUTCOMES?
}

\author{
Adrianna L. McIntyre \\ Mark Shepard \\ Myles Wagner \\ Working Paper 28630 \\ http://www.nber.org/papers/w28630 \\ NATIONAL BUREAU OF ECONOMIC RESEARCH \\ 1050 Massachusetts Avenue \\ Cambridge, MA 02138 \\ April 2021
}

This paper was prepared for an AER Papers \& Proceedings session presented in January 2021. We thank our discussant, Fiona Scott Morton, and Kate Ho, Marissa Woltmann, and Michael Norton for helpful comments. We thank the Massachusetts Health Connector for assistance in providing and interpreting the data. We gratefully acknowledge funding from Harvard's Lab for Economic Applications and Policy, Harvard Kennedy School's Rappaport Institute for Public Policy, and Harvard's Milton Fund. All errors are our own. The views expressed herein are those of the authors and do not necessarily reflect the views of the National Bureau of Economic Research.

NBER working papers are circulated for discussion and comment purposes. They have not been peer-reviewed or been subject to the review by the NBER Board of Directors that accompanies official NBER publications.

(C) 2021 by Adrianna L. McIntyre, Mark Shepard, and Myles Wagner. All rights reserved. Short sections of text, not to exceed two paragraphs, may be quoted without explicit permission provided that full credit, including ( $)$ notice, is given to the source. 
Can Automatic Retention Improve Health Insurance Market Outcomes?

Adrianna L. McIntyre, Mark Shepard, and Myles Wagner

NBER Working Paper No. 28630

April 2021

JEL No. D90,I13,I18

\section{ABSTRACT}

There is growing interest in market design using default rules and other choice architecture principles to steer consumers toward desirable outcomes. Using data from Massachusetts' health insurance exchange, we study an "automatic retention" policy intended to prevent coverage interruptions among low-income enrollees. Rather than disenroll people who lapse in paying premiums, the policy automatically switches them to an available free plan until they actively cancel or lose eligibility. We find that automatic retention has a sizable impact, switching $14 \%$ of consumers annually and differentially retaining healthy, low-cost individuals. The results illustrate the power of defaults to shape insurance coverage outcomes.

Adrianna L. McIntyre

Harvard University

amcintyre@g.harvard.edu

Mark Shepard

Harvard Kennedy School

Mailbox 114

79 JFK Street

Cambridge, MA 02138

and NBER

mark_shepard@hks.harvard.edu
Myles Wagner

Harvard University

mnwagner@g.harvard.edu 


\section{Introduction}

One of the best-established findings in behavioral economics is that people are often passive and that defaults - what happens when individuals fail to act - have a major impact on outcomes. There is growing interest in applying this principle to improve outcomes in policy-relevant settings.

Health insurance offers instructive examples. Programs like the Affordable Care Act's (ACA) exchanges aim to provide affordable coverage via markets that allow for choice and competition. However, these arrangements add complexity, and there is evidence that consumers struggle to choose well (Abaluck and Gruber, 2011; Bhargava et al., 2017) and exhibit inertia in plan switching decisions (Handel, 2013; Ericson, 2014; Ho et al., 2017). Default rules may therefore be quite impactful in these settings.

In this paper, we describe and evaluate a policy that leverages defaults to prevent loss of coverage when consumers lapse on premium payments, an important and under-appreciated challenge in health insurance. Under standard rules, lapsers are disenrolled, leaving them uninsured unless they obtain other coverage. We discuss an alternate policy, which we call "automatic retention," that instead defaults lapsers into a free plan if one is available.

We study auto retention empirically in Massachusetts' pre-ACA health insurance exchange, where the policy was used for several years with little attention. We are not aware of research that has described the policy or studied its effects.

We find that the policy has a major impact, retaining $14 \%$ of enrollees per year (weighted by duration enrolled). Auto retention is the primary way consumers switch plans, creating three times more switches than occur actively during open enrollment. The policy differentially retains young, healthy, and low-cost people, implying important consequences for the market's risk pool and extensive margin adverse selection. Using auxiliary data from the state's All-Payer Claims Database, we find little evidence that the policy leads to significant duplication of coverage. We conclude by discussing policy tradeoffs and implications.

\section{Exchange Background and the Auto Retention Policy}

Exchange Setting Our setting is Massachusetts' pre-ACA subsidized health insurance exchange, known as Commonwealth Care (“CommCare”). Established under the state’s 2006 "Romneycare” 
reform, CommCare provided subsidized private plans to low-income adults without access to insurance from an employer or public program. Subsidies were set to make the cheapest plan's premium "affordable," defined as $0-5 \%$ of monthly income. Additional background and statistics are discussed in Online Appendix A.

Like the ACA exchanges that followed it, CommCare took a regulated market-based approach. This structure puts policymakers in the position of market designers who set the rules under which insurers compete and consumers choose. Beyond standard “incentives” policies like subsidies and benefit regulation, market designers also devise rules related to what Thaler (2018) calls “choice architecture," which can have a large impact on boundedly rational consumers. Thoughtful choice architecture can “nudge” consumers towards desirable outcomes, while careless design can lead to poor outcomes. In this paper, we describe a nudge policy that affects what happens when consumers lapse on paying monthly premiums.

Challenge of Premium Lapses Most enrollees in health insurance markets owe some balance of monthly after-subsidy premiums. This raises the challenge of ensuring that consumers pay their bills. Premium lapses are common in health insurance exchanges. While we do not directly observe lapses in our CommCare data, $6 \%$ of consumers terminate enrollment each month. Data on subsidized enrollees in Massachusetts' post-ACA exchange (where reason for exit is observed) suggest that $30 \%$ of terminations are due to premium lapses.

The fundamental issue underlying lapsing is that the exchange has no way to automatically collect premiums. ${ }^{2}$ Consumers may opt out at any time, but what happens when they simply stop paying? Premium lapses create a dilemma for market designers. Should they disenroll the lapser which may lead to a spell of uninsurance and associated adverse consequences? Or should they weaken enforcement of premium collection? In practice, policymakers seek a balance, sending multiple notices over a grace period of 2-3 months before disenrolling a lapser. However, more creative approaches may be desirable to improve on this outcome.

\footnotetext{
${ }^{2}$ As such, an alternate approach would be to find a way to automatically collect or withhold premiums, possibly via the tax system. This approach is used successfully by both employers (withholding from paychecks) and Medicare (withholding from Social Security benefits). Auto-collection via taxes was not feasible in CommCare due to crossdepartment legal and administrative barriers. But tax-based collection seems a natural fit for the ACA, whose subsidies are administered by the IRS as income tax credits.
} 
Automatic Retention Policy Auto retention was an approach to reduce coverage interruptions for lapsers. Rather than automatically disenroll premium lapsers, the policy instead automatically switched them to a \$0 premium plan if available. Lapsers carried debt for unpaid premiums but retained coverage unless they actively canceled or lost eligibility. If they paid this debt within 60 days, they could switch back to their old plan.

The key precondition for auto retention is the availability of "backstop" coverage that is free (or more generally, in which up-front premium collection can be waived). In CommCare, this condition held only for the $100-150 \%$ of poverty income group, for whom the cheapest plan was free while other plans varied from \$2 to \$34 per month. Auto retention was not used for higherincome groups who did not have access to a $\$ 0$ plan. ${ }^{3}$ We use the $150-200 \%$ of poverty group (for whom the cheapest plan costs $\$ 39-40$ ) as a control group in our analysis.

\section{Data and Methods}

Our main dataset is de-identified CommCare enrollment records linked to insurer claims. Online Appendix A describes the dataset and cleaning process. We limit our analysis to fiscal years 20102013, when the auto retention policy was in place. ${ }^{4}$

A limitation of our data is that they do not include an indicator for plan switching due to auto retention. We infer its use from the (much higher) rates of “mid-year” plan switching for the 100$150 \%$ of poverty group. We first drop a small number of known cases where mid-year switching is allowed (changes in service area or income group). To proxy for harder-to-observe exceptions, we use mid-year switching rates for the 150-200\% "control” group. ${ }^{5}$ Our estimate of the rate of auto retention is the excess mid-year switching rate for the "treatment" group (100-150\% of poverty) relative to the controls.

A second limitation of the CommCare data is that it lacks information on other sources of health insurance. To assess whether auto retention leads to duplicate coverage, we draw on

\footnotetext{
${ }^{3}$ Auto retention was unnecessary for below-poverty enrollees who had access to all plans for free, making premium lapsing moot.

${ }^{4}$ Auto retention appears to have been used inconsistently in 2009, so we exclude it for simplicity. It was not used prior to 2009 , since all plans were free for the $100-150 \%$ of poverty group.

${ }^{5}$ Other exceptions include the dropping of an enrollee's PCP from network and receipt of a special hardship waiver. In practice, these appear to be rare; the control group’s mid-year switching rates are less than $0.3 \%$ per month.
} 
Massachusetts' All-Payer Claims Database (APCD), which lets us observe enrollment in both CommCare and nearly all other health insurance in the state. Online Appendix A further describes our APCD cleaning methods.

\section{Results}

\subsection{Auto Retention Estimates}

Figure 1 shows the switching patterns underlying our estimates. The panels show monthly plan switching rates during 2010-13 for the treatment and control groups (with 2010-11 pooled because patterns are similar). Open enrollment switches in the first month of the year (shaded in gray) are excluded from our estimates but shown for context.

Two results stand out in Figure 1. First, mid-year switching rates are an order of magnitude higher for the treatment group (averaging 2.2\% per month) compared to the control group ( $0.24 \%$ per month). The excess switching rate- our estimate of the impact of auto retention - is $1.9 \%$ per month, on average. When summed over all 11 mid-year months, auto retention results in about three times more switches than occurs during formal open enrollment (which averages 6-7\% for one month). Automatic retention is the primary way consumers switch plans in the treatment group.

To translate these monthly rates into annual estimates, we calculate the share of total enrolleemonths accounted for by mid-year switchers in each year. This share is $15.3 \%$ in the treatment group and $1.5 \%$ in the control group, implying an excess share of $13.8 \%$. This is our main estimate of the share of consumers affected by auto retention. ${ }^{6}$

The second clear pattern in Figure 1 is a large switching spike in months 3 or 4 of each year except 2012. Excess switching rates average 9.3\% during these spikes, versus $1.4 \%$ in all other months. This appears to be driven by changes in which plans are free at the start of the year. When a plan shifts from free to non-free, its enrollees face a choice to either: (1) actively switch to a different plan that is now free, or (2) stick with their current plan and actively pay a premium. In

\footnotetext{
${ }^{6}$ This estimate of $13.8 \%$ is lower than 11 times the monthly excess switching rate (1.9\%) because of consumer churn into and out of the sample. See Online Appendix Table B.1 for these statistics.
} 
practice, many enrollees do neither, instead lapsing. This results in an auto-switching spike just after the 2-3-month grace period ends.

Figure 2 shows evidence for this interpretation. It breaks down treatment group switching rates by the origin plan's free/non-free status in the current and prior year. ${ }^{7}$ Only plans that shift from being free to non-free (blue series) show a spike. Plans that remain non-free in both years (red series) exhibit steady mid-year switching but no spike. Switching out of free plans (green series) is much lower; this is expected, as one cannot lapse on a \$0 premium. This story also explains why there was no switching spike for 2012 in Figure 1; this was the only year that the prior year's free plan remained free. ${ }^{8}$ These patterns suggest that plan transitions from free to nonfree are an important trigger for lapsing and may merit attention by policymakers.

\subsection{Mechanisms: Financial vs. Hassle Costs}

Why do so many enrollees lapse on paying premiums? While the reasons are undoubtedly complex, one key question is whether lapsing reflects the financial cost (or "affordability") of a higher premium or the hassle cost of paying any positive premium (e.g., the time and attention cost of remembering to pay the bill)? These stories have different policy implications so are worth distinguishing.

To do so, we explore the relationship between mid-year switching rates and the premium of the origin plan (see Online Appendix Figure B.3). Our analysis suggests a role for both mechanisms. Hassle costs appear to be key during the month 3-4 switching spike. There is little relationship between origin plan premium and auto-switching rates, and they are high even in cases with very low premiums ( $<\$ 5$ per month). One example is illustrative: a plan whose premium increased from $\$ 0$ to $\$ 3$ at the start of 2013. Following this change, 24\% of its enrollees autoswitch out in 2013m3, and another $2.5 \%$ per month switch out during the rest of the year. It is implausible that \$3 per month is unaffordable; instead, this must reflect some form of hassle cost.

\footnotetext{
${ }^{7}$ Figure 2 pools estimates for 2010-12 and omits 2013 because of the different spike timing in 2013 (month 3 rather 4). CommCare updated regulations at this time, limiting the grace period to 2 months starting in 2013. Online Appendix Figure B.2 shows estimates for each year 2010-13, which are similar to the pooled results.

8 This is true statewide except for one small area in Western Massachusetts where (because of an insurer entry) the free plan in 2011 became non-free in 2012. Consistent with our story, we see a large (19.0\%) switching spike in this region only (see Online Appendix Figure B.2). Because the area is small, it is not visible in Figure 1.
} 
We do, however, see evidence for financial costs mattering outside of the month 3-4 spike. For these months, we find that an additional \$10 per month premium obligation raises the midyear switching rate by $0.5-1.0 \%$ points (relative to an average of $1.8 \%$ ). Although $\$ 10$ is a modest amount - just $1 \%$ of monthly income even at the poverty line - this analysis shows that even nominal premiums can deter enrollment in low-income groups.

\subsection{Heterogeneity Analysis}

The auto retention policy differentially affects certain groups. Online Appendix Table B.2 compares mid-year switchers (a proxy for auto-retained enrollees) to all other enrollees in the treatment group. Switchers are younger (by 4.1 years), less likely to have a chronic illness (by $3.4 \%$ points, or $6 \%$ ), and have lower medical risk scores (by 0.025 , or $2.5 \%$ lower predicted spending). Their average medical spending per month enrolled is $8.6 \%$ lower. Notably, the larger percentage gap in spending than risk score indicates that switchers are differentially profitable even after risk adjustment. Spending for auto-switchers is particularly low in the six months following the auto-switch, consistent with research showing that enrollees lapse at times when they use less health care (Diamond et al., 2020).

The average switcher stays enrolled in CommCare for 10 months after the switch, which is substantial in a market where typical durations are about a year. Notably, 15\% of switchers "reswitch" within three months of their auto-switch. This is non-trivial, but implies that the vast majority (85\%) stick with their newly assigned plan, boosting the market share of these lowestprice plans.

\subsection{Does Auto Retention Lead to Duplicate Coverage?}

A key concern with auto retention is that it retains enrollees who may have gained other insurance (e.g., via a new job) and should technically be ineligible for CommCare. ${ }^{9}$ Duplicative coverage would not harm enrollees but would result in unneeded public spending on subsidies. Using the

\footnotetext{
9 The exchange attempts to avoid duplication via a unified Medicaid-CommCare enrollment system (which should mechanically prevent inappropriate duplication), annual eligibility redetermination, and periodic cross-checks of enrollee lists for commercial insurance. However, these safeguards may still miss some enrollees.
} 
APCD, however, we find that coverage duplication rates for CommCare enrollees are low (3.1\%) and not much different for enrollees in the 11 months surrounding a mid-year plan switch (3.6\%).

\section{Discussion}

This paper has described a policy we call "automatic retention," which Massachusetts used in its pre-ACA insurance exchange to reduce termination for premium non-payment among low-income health insurance enrollees. Rather than disenrolling lapsers, the policy automatically switched them to a free plan if one is available. Our analysis suggests the policy had a major impact, retaining $14 \%$ of consumers per year. Retained enrollees are younger, healthier, and lower-cost, suggesting that the policy improves the market risk pool. We were concerned auto retention would lead to duplicate coverage, but evidence from the APCD suggests duplication is rare and not much different for enrollees around the time of mid-year plan switches.

A limitation of our analysis is that we do not see counterfactual outcomes for the $100-150 \%$ of poverty treatment group without auto retention in place. Absent auto retention, we expect that lapses would mechanically lead to termination, but we do not know how transient or long-lasting the coverage gap would be. In separate work on the post-ACA Massachusetts exchange (when auto retention was no longer in effect), McIntyre (2020) finds that changes in which plans were free/non-free lead to a large spike in terminations due to non-payment for the same $100-150 \%$ of

poverty group. The vast majority of terminated consumers do not return within 12 months, suggesting that coverage gaps may be significant.

The finding that defaults matter for retaining enrollees in health insurance adds to a broader literature on the power of defaults to shape market outcomes. Most prior work on defaults within health insurance has focused on consumer inertia when given an opportunity to switch plans. In ongoing work on the same Massachusetts market, two of us also find large impacts of an automatic enrollment default during the initial sign-up process (Shepard and Wagner, 2021).

Our findings point to a key role for the hassle cost of paying a premium in driving lapses, rather than affordability. "Hassles" may reflect a variety of factors, including informational barriers (e.g., lost or unopened mail notices), the time cost of setting up online auto-payment, or the attention cost of remembering to write a check each month. Further research into mechanisms would be useful in guiding policy responses. Finding a way to withhold or collect premiums 
automatically - a strategy used successfully by employers and Medicare - would address many of these issues.

There are tradeoffs inherent to auto retention. In reducing terminations, the policy increases subsidized insurance enrollment. On the one hand, reducing uninsurance is a key policy goal. On the other hand, public subsidy spending also rises. Whether that spending is "worth it," given benefits to the newly insured and spillover benefits to society, is a key issue animating current debate about the ACA.

Another tradeoff involves the policy's effect on competitive incentives. The policy boosts market share for the lowest-price plan(s) that receive auto-switched individuals. This should encourage insurers to compete aggressively to be the lowest-price plan. However, this price competition could lead to quality reductions and may be distorted by risk selection incentives. Like other policies, auto retention appears to involve a tradeoff between improving risk selection on the extensive margin while worsening it on the intensive margin (Saltzman 2020; Geruso et. al, 2020).

Implementation of auto retention in other settings, like the ACA exchanges, would face similar tradeoffs, in addition to legal and practical challenges. Nonetheless, our evidence suggests that if these challenges could be surmounted, changing default rules can meaningfully improve coverage retention.

\section{References}

Abaluck, Jason, and Jonathan Gruber. 2011. "Choice inconsistencies among the elderly: evidence from plan choice in the Medicare Part D program.” American Economic Review 101, no. 4: 1180-1210.

Bhargava, Saurabh, George Loewenstein, and Justin Sydnor. 2017. "Choose to lose: Health plan choices from a menu with dominated option.” The Quarterly Journal of Economics 132, no. 3: 13191372.

Chandra, Amitabh, Jonathan Gruber, and Robin McKnight. 2014. "The impact of patient cost-sharing on low-income populations: evidence from Massachusetts.” Journal of health economics 33: 57-66.

Diamond, Rebecca, Michael J. Dickstein, Timothy McQuade, and Petra Persson. 2020. "Insurance without Commitment: Evidence from the ACA Marketplaces.” Working paper.

Ericson, Keith M. 2014. "Consumer inertia and firm pricing in the Medicare Part D prescription drug insurance exchange.” American Economic Journal: Economic Policy 6, no. 1: 38-64.

Finkelstein, Amy, Nathaniel Hendren, and Mark Shepard. 2019. "Subsidizing health insurance for lowincome adults: Evidence from Massachusetts.” American Economic Review 109, no. 4: 1530-67.

Handel, Benjamin R. 2013. "Adverse selection and inertia in health insurance markets: When nudging 
hurts.” American Economic Review 103, no. 7: 2643-82.

Ho, Kate, Joseph Hogan, and Fiona Scott Morton. 2017. "The impact of consumer inattention on insurer pricing in the Medicare Part D program.” The RAND Journal of Economics 48, no. 4: 877-905.

Geruso, Michael, Timothy J. Layton, Grace McCormack, and Mark Shepard. 2019. "The two margin problem in insurance markets” No. w26288. National Bureau of Economic Research.

McIntyre, Adrianna. 2020. "Small Premiums or Big Administrative Burdens? Nominal Contributions and Disenrollment in the Massachusetts Nongroup Market.” Working paper.

Saltzman, Evan. 2018. “Managing Adverse Selection: Underinsurance vs. Underenrollment.” Working paper.

Shepard, Mark. 2016. "Hospital network competition and adverse selection: evidence from the Massachusetts health insurance exchange.” No. w22600. National Bureau of Economic Research.

Shepard, Mark and Myles Wagner. 2021. “The Economics of Automatic Health Insurance Enrollment.” Working paper.

Thaler, Richard H. 2018. "From cashews to nudges: The evolution of behavioral economics." American Economic Review 108, no. 6: 1265-87. 
Figure 1: Share of Enrollees Switching Plans, by Month

Panel A: 2010-2011 (pooled)

Panel B: 2012
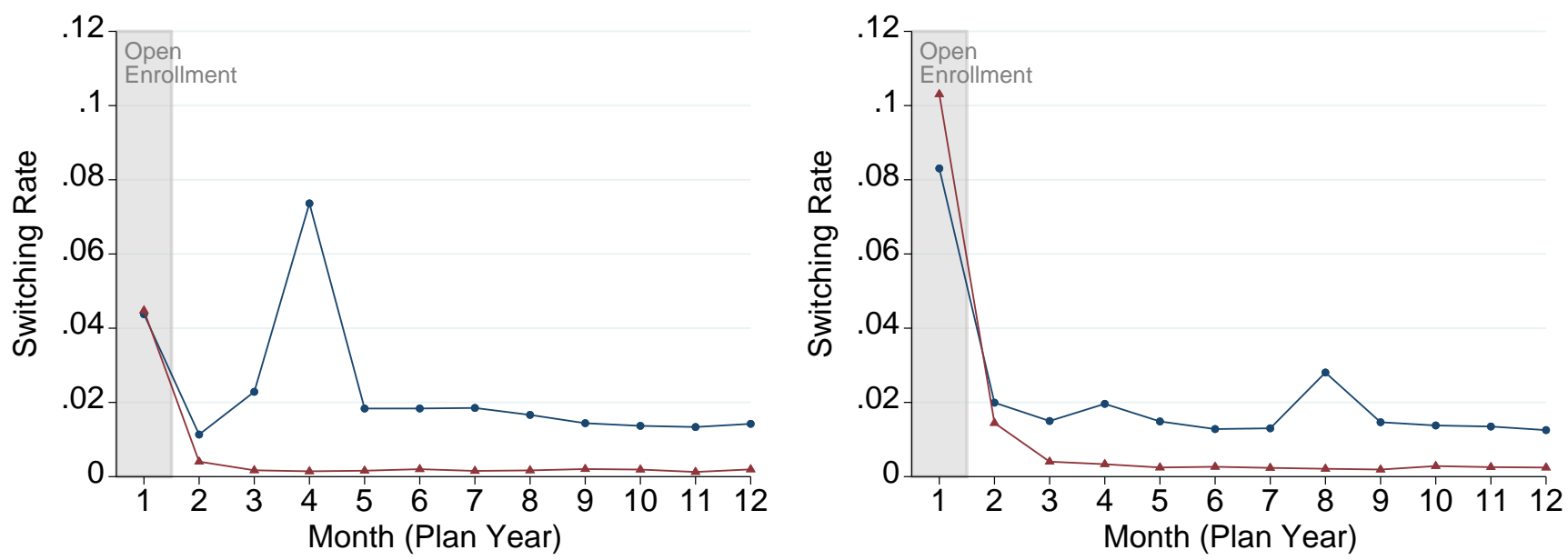

Panel C: 2013

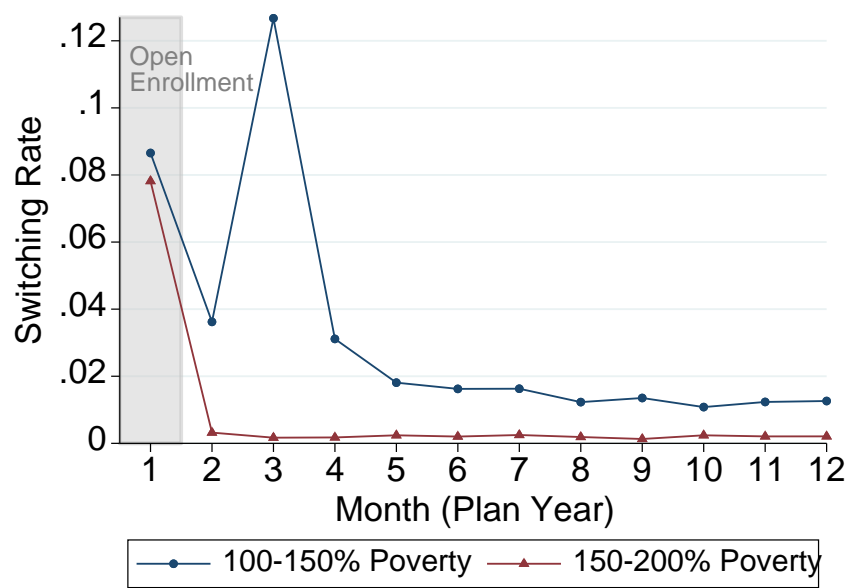

NOTE: The figure shows the share of sample enrollees who switch plans by month of the year for the treatment group subject to auto retention (100-150\% of poverty, in blue) and control group not subject to the policy (150-200\% of poverty, in red). Panel A shows 2010-11 (pooled because of similar patterns); panel B shows 2012; and panel C shows 2013. Open enrollment, when switching is typically allowed, is shaded in gray. Higher switching rates in all other (“mid-year") months for the treatment group indicate the impact of the auto retention policy. 
Figure 2: Plan Switching Rates, by Origin Plan Free/Non-free Status

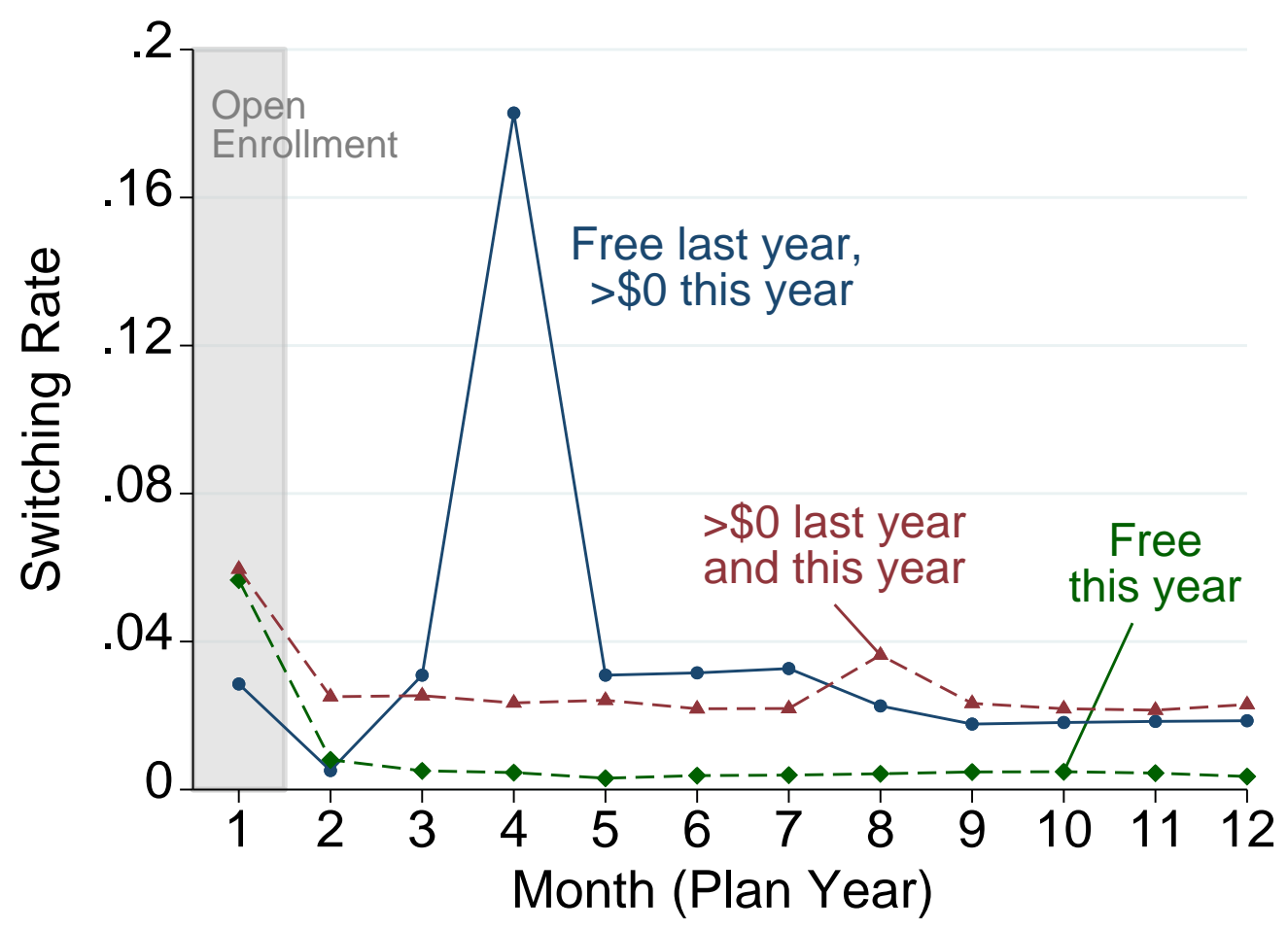

NOTE: The figure breaks down switching rates for the treatment group (100-150\% of poverty) by the free/non-free status of the origin plan to understand the source of the large switching spike in Figure 1. It shows monthly switching rates out of three types of plans: (1) plans that were free last year but become non-free this year (blue solid line), (2) plans that were non-free $(>\$ 0)$ both last year and this year (red dashed), and (3) plans that are free this year, regardless of their premium last year. Statistics are pooled across 2010-2012 for simplicity, with 2013 omitted because of its different timing of the spike (month 3 rather than month 4). Results are similar if broken down separately by year (see Appendix Figure B.2). The figure indicates that all of the large switching spike comes from enrollees in plans that change from being free to non-free at the start of the new year. 


\section{Online Appendix:}

\section{“Can Automatic Retention Improve Health Insurance Market Outcomes?”}

By Adrianna McIntyre, Mark Shepard, Myles Wagner

\section{Appendix A: Data, Sample, and Methods Details}

CommCare Dataset Our main dataset is complete (de-identified) administrative enrollment data for the CommCare program, linked to insurer claims. This data was obtained via a data use agreement with the Massachusetts Health Connector, the administrator of CommCare. We thank the Connector for its assistance in providing and interpreting the data. The dataset is structured at the enrollee $\mathrm{x}$ month level, with information on individual income group, location, demographics, and plan enrollment during that month. To this, we merge on information on monthly spending and (at an annual level) medical diagnoses and risk scores calculated from the linked claims dataset.

Starting from the full data that spans fiscal years 2007-2014, we limit our sample to the fiscal year 2010-13 period when the auto retention policy was consistently in effect. ${ }^{10}$ (CommCare's fiscal year runs from July-June, so this represents in calendar time July 2009 to June 2013.) We next limit the sample to enrollee-month observations in either the $100-150 \%$ of poverty "treatment" group (subject to auto retention) or the 150-200\% of poverty "control” group (not subject to the policy).

Sample Limitations for Measuring Auto Switching Rate Our goal is to measure the rate of automatic plan switching due to the automatic retention policy. However, a key limitation of the data is that they do not include a direct flag for plan switching due to the policy. Instead, we infer its use from the (much higher) rates of "mid-year” plan switching - outside of the beginning-

${ }^{10}$ We make these drops for the following reasons. The auto retention policy was not in effect in 2007 (when the cheapest plan was not free for the treatment group) or 2008 (when all plans were free for the treatment group, making lapsing irrelevant). We exclude 2009 because auto retention appears to have been used inconsistently during the year and because there was an extended open enrollment period (months 1-3) that makes it difficult to separate out automatic from active switching. We exclude fiscal 2014 both because is a short year (July 2013 to December 2013) and because its latter months interact with the implementation of the ACA. 
of-year open enrollment period when switching is allowed - for the $100-150 \%$ of poverty group versus other groups.

Aside from open enrollment, there are a few exceptions that allow for mid-year switching. We observe and exclude from our sample instances of the two main exceptions: changes in geographic area or income group. These exclusions - which we make whether or not the enrollee actually switched plans - drop 0.4\% (geography changes) and 3.2\% (income changes) of member-month observations, of which only $1.6 \%$ represent switches. Enrollees are also technically allowed to switch plans within two months of initially enrolling in the market. We chose not to drop these cases because they represent a relatively large share of the sample (12\%) and switching rates are not much higher in these months, even in the control group. Enrollees are also allowed to "reswitch” plans in the 60 days following an auto-switch; we retain these observations in the sample because this is an outcome of interest.

Table A.1 shows summary statistics for the final sample, separately for the treatment (100$150 \%$ of poverty) and control (150-200\% of poverty) groups. The two groups face different premiums - e.g., the cheapest plan is \$0 in the treatment group versus \$39-40 in the control group - which leads to differential selection into participation in the market (Finkelstein, Hendren, and Shepard, 2019). This selection can account for some of the age and medical spending differences for the two groups. These differences should not have a major impact on mid-year plan switching rates aside from the auto retention policy. Moreover, the mid-year switching rate is so low for the control group $(0.2 \%)$, that all of our results would be similar if we simply analyze patterns for the treatment group.

Measuring Switching and Excess Plan Switching Rates The key variable for our analysis is the plan switching rate, defined as the share of individuals continuously enrolled between months $t-1$ and $t$ who switch plans between those months. We calculate switching rates both for the open enrollment period (month 1) when active switching is allowed for any reason, and for months 2-12 of the year ("mid-year" months) when active switching is typically not allowed.

As described above, we drop observations with known exceptions that allow for mid-year switching (changes in income group or service area). In addition, an individual who has lapsed and been auto-switched may "re-switch" back to their old plan within 3 months if they pay their

premium debt. To avoid counting re-switches in our estimates of auto retention (e.g., for Figure 
1), we do not count mid-year switches within 3 months of another plan switch for either the treatment or control group. (These observations are retained in the sample but recoded as nonswitches for calculating the switching rate.) We instead report re-switching as a separate outcome in Table B.2.

During open enrollment, switching rates are similar for the treatment and control groups (6.5\% and 6.9\%, respectively). However, "mid-year" switching rates in the other 11 months are an order of magnitude higher for the treatment group (2.2\% per month) relative to the control group (0.24\%). This is consistent with our understanding of the data generating process. The low control group switching rate reflects a few hard-to-observe exceptions that allow for mid-year switching, while the much higher rate for the treatment group reflects the auto retention policy.

To measure the excess switching rate formally, we run the following OLS regression:

$$
1\left\{\text { SwitchPlans }_{i t}\right\}=\alpha_{t}+\beta \cdot 1\left\{\text { TreatGrp }_{i t}\right\}+\varepsilon_{i t}
$$

where $\alpha_{t}$ are time fixed effects that capture baseline switching rates in the control group, and $\beta$ (the coefficient of interest) captures the excess switching rate for the treatment group. In some analyses, we run regression (1) separately for certain subsets of plans (e.g., plans that transition from free to non-free) or subsets of time periods (spike month vs. non-spike months).

Supplementary Dataset: Mass. APCD A key limitation of the CommCare data is that we cannot observe insurance outside of the CommCare market. A question of particular interest is whether the auto retention policy leads to duplicate coverage in both CommCare and outside private insurance. To assess this, we draw on information from the Massachusetts All-Payer Claims Database (APCD). ${ }^{11}$ The APCD lets us observe coverage in both CommCare and nearly all other health insurance in the state - with the sole important exception being traditional Medicare, which is unlikely to be relevant for the non-elderly, non-disabled population in CommCare. The APCD includes a synthetic ID that follows individuals across insurers, letting us observe duplicate coverage.

Using the APCD's member eligibility file, we construct an enrollment history dataset for people ever enrolled in CommCare that also includes their coverage history in other insurance.

\footnotetext{
${ }^{11}$ We use the APCD version 3.0, which includes calendar years 2009-2013. The APCD, which is not linked to the CommCare data, was obtained under a separate data use agreement with Massachusetts' Center for Health Information and Analysis.
} 
The data construction requires some care. Each record in the ME file describes a member's enrollment spell in a particular health plan, with variables describing the characteristics of the health plan (such as the plan's carrier), and the start- and end-dates of the spell. We use the variables "Insurance Type Code” (ME003) and "Special Coverage” (ME031) as CommCare indicators. Both are categorical variables that indicate a CommCare enrollment; however, since they do not always coincide, we define our sample of CommCare enrollment spells as those for which either variable indicates CommCare.

An additional challenge is that many records for BMC enrollments have missing values for the end-date, specifically coded as "12/31/2099" or "12/31/2199." We find that these are often (in about $98 \%$ of cases) accompanied by another record with an identical start-date and a non-missing end-date. In these cases, we disregard the record with the missing end-date in the construction of our panel. In the remaining $2 \%$ of cases, we truncate the end-date to be $12 / 31 / Y Y Y Y$, where YYYY is the year of the report ("eligibility year", given by the variable ME004).

We validate the construction of this dataset by comparing it to the true CommCare enrollment data. The numbers line up quite closely. The APCD CommCare subset matches within $3 \%$ the member-month counts in the true CommCare data for fiscal years 2009-2013 (10.7 million in the APCD compared to 10.4 million true CommCare member-months). Enrollment across plans and over time also line up quite closely.

With this panel dataset in hand, we turn to non-CommCare enrollment spells in the APCD. We do not have an external dataset to validate the non-CommCare enrollment, so we take the spell descriptors in the APCD at face-value. We define dual enrollment as a month in which a CommCare member is also enrolled in non-CommCare health insurance. 
Table A.1: Sample Summary Statistics

\begin{tabular}{|c|c|c|}
\hline & $\begin{array}{c}\text { 100-150\% Poverty } \\
\text { (Treatment Grp.) } \\
\text { (1) }\end{array}$ & $\begin{array}{c}\text { 150-200\% Poverty } \\
\text { (Control Grp.) } \\
\text { (2) }\end{array}$ \\
\hline \multicolumn{3}{|l|}{ Enrollment and Switching } \\
\hline Total enrollment per month & 35,108 & 28,067 \\
\hline Terminations per month & 1,811 & 1,752 \\
\hline $\begin{array}{l}\text { Duration Enrolled (months): Median } \\
\text {. }\end{array}$ & 14.0 & 13.0 \\
\hline Mean & 19.7 & 18.0 \\
\hline \multirow[t]{2}{*}{ Share Switch Plans: } & $6.53 \%$ & $6.87 \%$ \\
\hline & $2.17 \%$ & $0.24 \%$ \\
\hline \multicolumn{3}{|c|}{ Consumer Premiums (\$/month, after subsidies) } \\
\hline Lowest-Premium Plan & $\$ 0.00$ & $\$ 39.29$ \\
\hline \multirow[t]{2}{*}{ Other Plans: } & $\$ 8.03$ & $\$ 53.85$ \\
\hline & $\$ 34.00$ & $\$ 91.00$ \\
\hline \multicolumn{3}{|l|}{ Consumer Attributes and Costs } \\
\hline Age (years) & 42.6 & 44.6 \\
\hline Share Male & $42.1 \%$ & $40.6 \%$ \\
\hline Income (\% of Poverty Line) & 127.5 & 174.5 \\
\hline Risk Score (HHS-HCC) & 1.037 & 1.128 \\
\hline Medical Costs (\$ per month) & $\$ 334.70$ & $\$ 376.80$ \\
\hline
\end{tabular}

NOTE: The table shows summary statistics for our sample, separately for the treatment group subject to the auto retention policy (100-150\% of poverty) and the control group not subject to the policy (150-200\% of poverty). Risk score (HHS-HCC) refers to the HHS Hierarchical Condition Category risk adjustment method used in the ACA Marketplaces. We impute this risk score for each enrollee at an annual level based on demographics and diagnoses observed on their claims and normalize mean risk score to 1.0 for the whole market. Medical costs refer to average monthly medical spending (insurer-paid and cost sharing) during the enrollment spell. 


\section{Appendix B: Additional Figures and Tables}

Figure B.1: Share of Enrollees Switching Plans, by Month (single-year version of Figure 1 in text)

Panel A: 2010

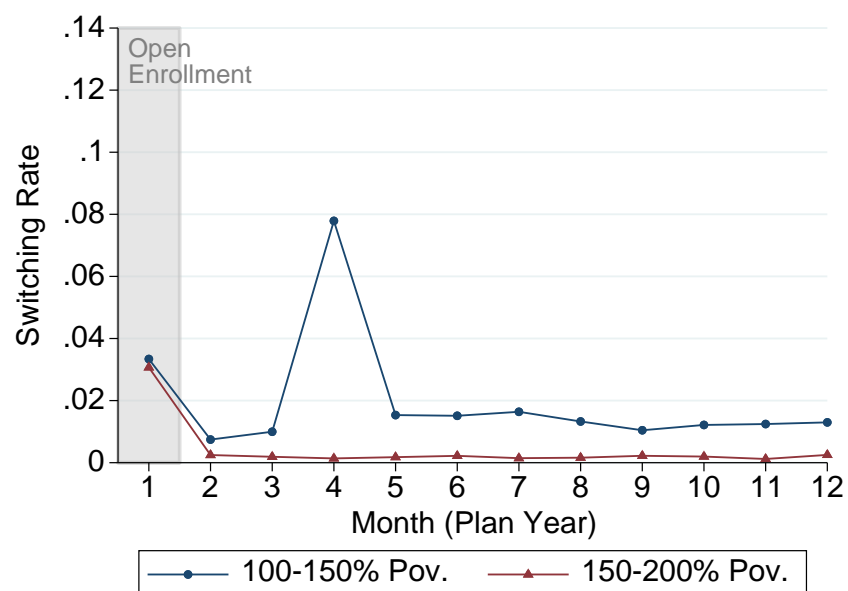

Panel C: 2012

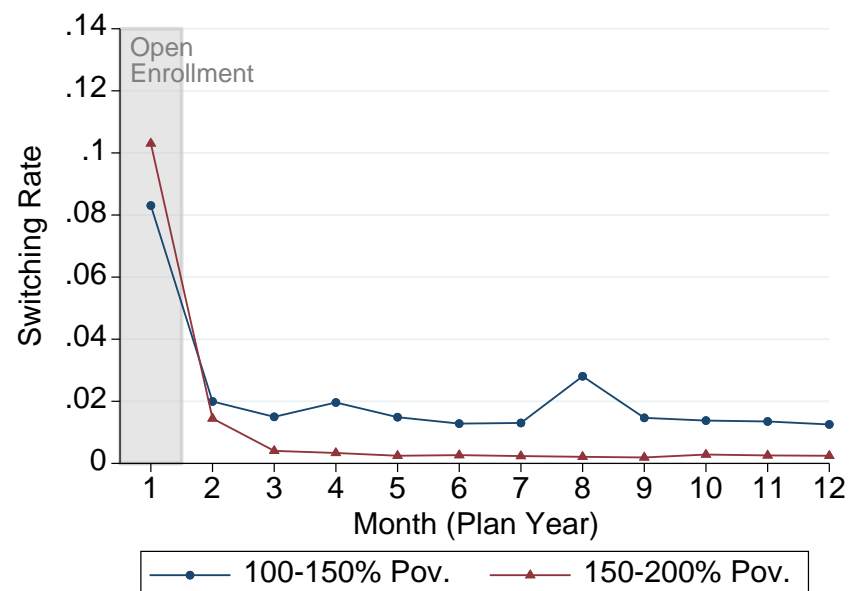

Panel B: 2011

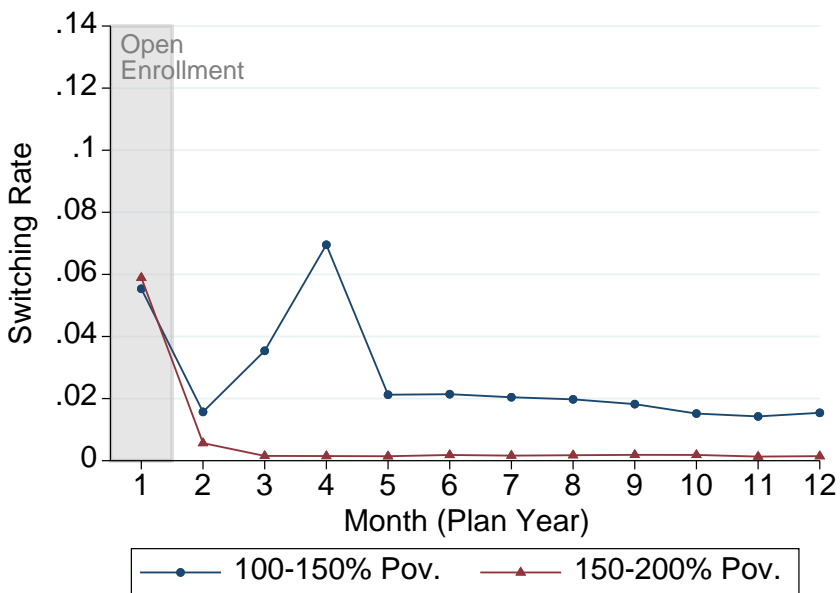

Panel D: 2013

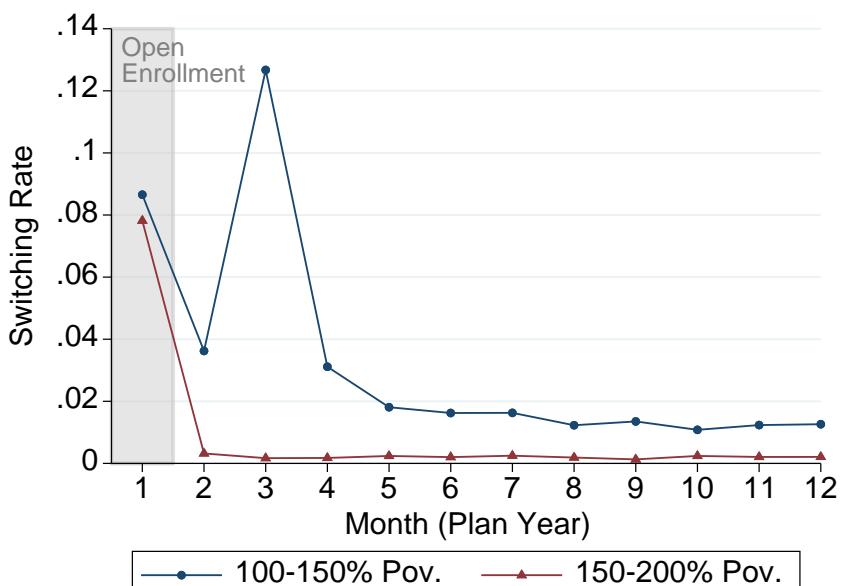

NOTE: The figure shows an annual version of Figure 1 in the body text for each year 2010-13. The figure shows the share of sample enrollees who switch plans by month of the year for the treatment group subject to auto retention (100-150\% of poverty, in blue) and control group not subject to the policy (150-200\% of poverty, in red). Open enrollment, when switching is typically allowed, is shaded in gray. Higher switching rates in all other ("mid-year") months for the treatment group indicate the impact of the auto retention policy. 
Figure B.2: Plan Switching Rates, by Origin Plan Free/Non-free Status

(single-year version of Figure 2 in text)

Panel A: 2010

Panel B: 2011
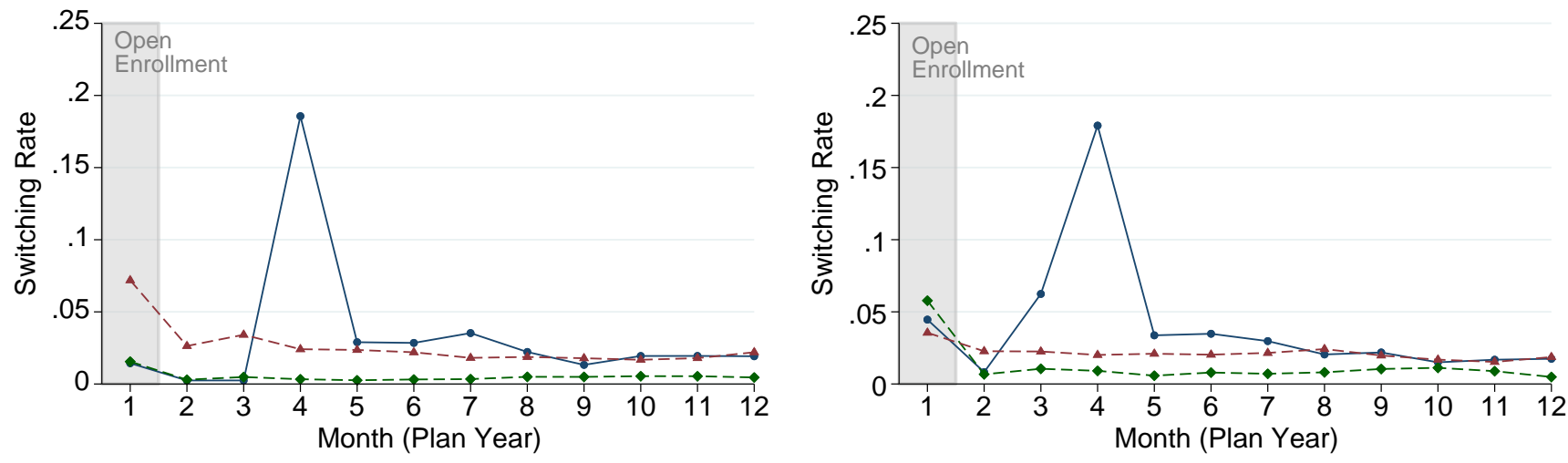

$\longrightarrow$ Free last yr, $>\$ 0$ this yr $\ldots-$ Free this year $-->\$ 0$ this and last yr

Panel C: 2012

Panel D: 2013
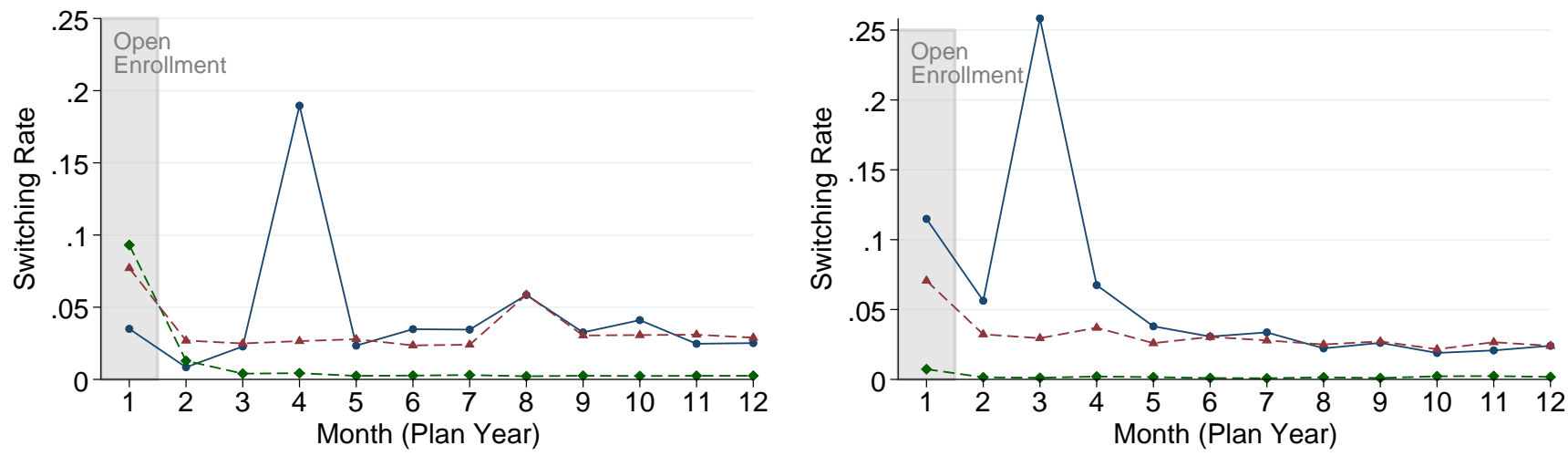

$\longrightarrow$ Free last yr, $>\$ 0$ this yr $\cdots$ Free this year $-->\$ 0$ this and last $\mathrm{yr}$

$\longrightarrow$ Free last yr, $>\$ 0$ this yr $-\cdots$ Free this year $-->\$ 0$ this and last $y r$

NOTE: The figure shows an annual version of Figure 2 in the body text for each year 2010-13. The figure breaks down switching rates for the treatment group (100-150\% of poverty) by the free/non-free status of the origin plan to understand the source of the large switching spike in Figure 1. It shows monthly switching rates out of three types of plans: (1) plans that were free last year but become non-free this year (blue solid line), (2) plans that were non-free (>\$0) both last year and this year (red dashed), and (3) plans that are free this year, regardless of their premium last year. Consistent with the results in Figure 2, these figures indicate that all of the large switching spike in month 3 or 4 comes from enrollees in plans that change from being free to non-free at the start of the new year. 
Figure B.3: Mid-Year Switching Rates vs. Origin Plan Premium Amount

Panel A: Prior-Year Free Plans

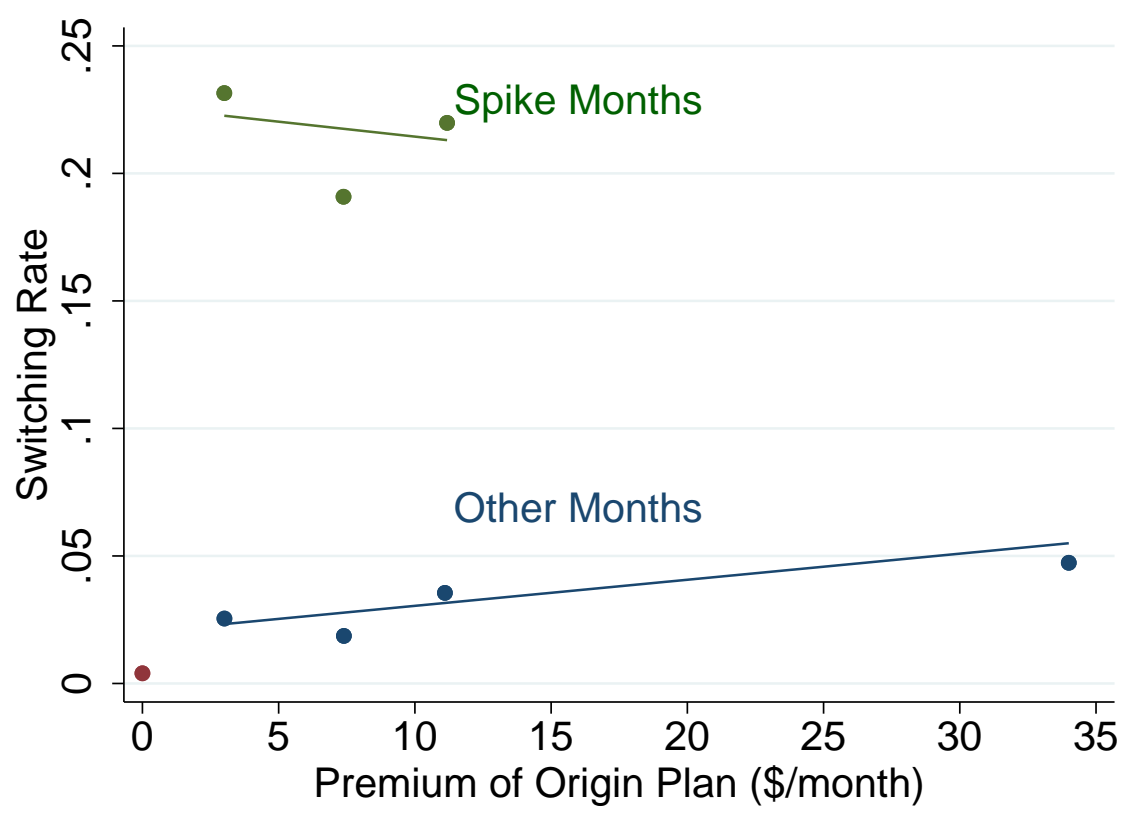

Panel B: Prior-Year Non-Free Plans

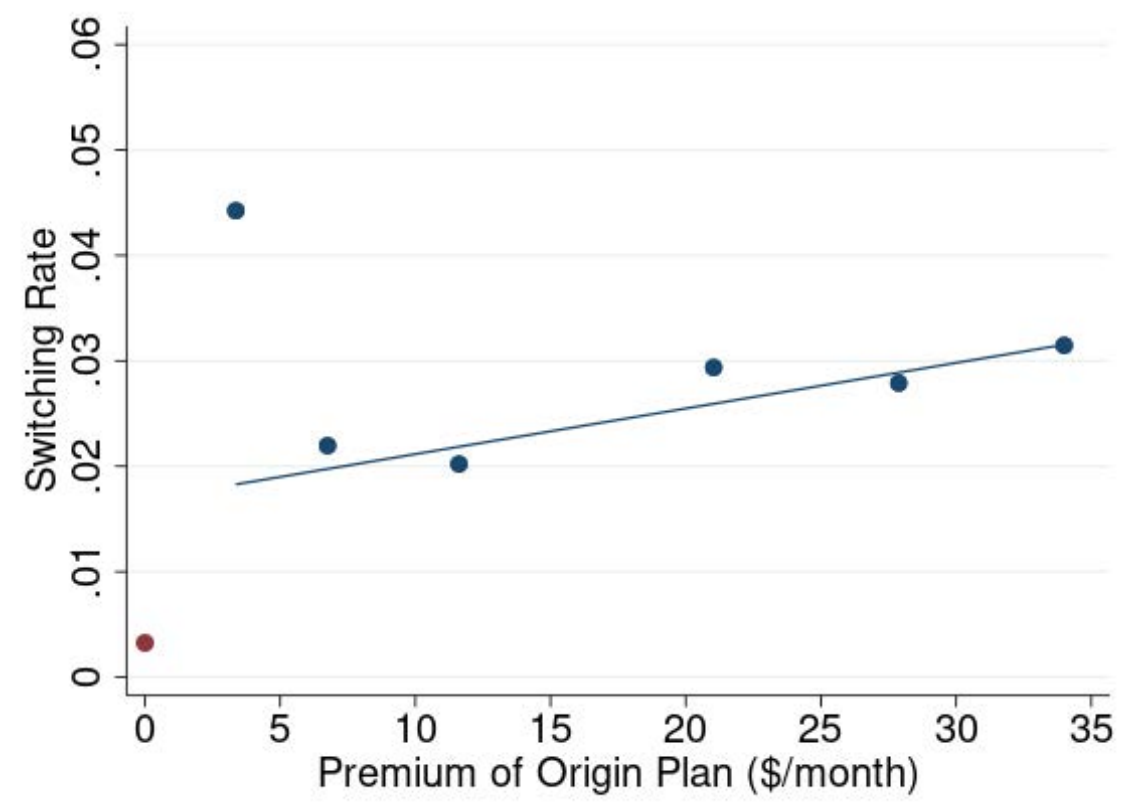

NOTE: The figure shows binned scatter plots of the relationship between mid-year switching rates and the monthly premium of the origin plan during the current year. In all cases, $\$ 0$ current premium is included as a separate category (shown in red). Based on the patterns in Figure 2, the relationship is broken down by plans that were free in the prior year (panel A) versus non-free plans in the prior year (panel B). Panel A is further broken down between the spike months (month 3 or 4, depending on the year) and non-spike months. 
Table B.1: Prevalence of Mid-Year Switchers, Treatment vs. Control

\begin{tabular}{|c|c|c|}
\hline & $\begin{array}{c}\text { 100-150\% Poverty } \\
\text { (Treatment Grp.) } \\
\text { (1) }\end{array}$ & $\begin{array}{c}\text { 150-200\% Poverty } \\
\text { (Control Grp.) } \\
(2)\end{array}$ \\
\hline Share of member-years w/ mid-year switch & $11.96 \%$ & $1.32 \%$ \\
\hline Share, weighted by months enrolled in yr. & $15.27 \%$ & $1.51 \%$ \\
\hline Avg. Members per Year & 66,582 & 55,643 \\
\hline
\end{tabular}

NOTE: The table shows the share of member-years from the pooled 2010-2013 sample that experience at least one mid-year switch, for the $100-150 \%$ of poverty group (column 1) and the $150-200 \%$ of poverty group (column 2 ), both un-weighted and weighted by the number of months observed in each year (excluding months with a change in geographic area or income group).

Table B.2: Characteristics of Mid-Year Switchers vs. Other Enrollees

\begin{tabular}{|c|c|c|c|c|c|}
\hline & \multicolumn{5}{|c|}{ 100-150\% Poverty Enrollees } \\
\hline & $\begin{array}{c}\text { Mid-Year } \\
\text { Switchers } \\
(1)\end{array}$ & $\begin{array}{c}\text { All Others } \\
\text { (2) }\end{array}$ & Diff. & $\begin{array}{l}\text { (s.e.) } \\
\text { (3) }\end{array}$ & $\begin{array}{c}\text { Combined } \\
\text { Population } \\
(4)\end{array}$ \\
\hline Share of Enrollment Months & $15.3 \%$ & $84.7 \%$ & -- & & $100 \%$ \\
\hline \multicolumn{6}{|l|}{ Demographics and Risk } \\
\hline Income (\% of Poverty Line) & 126.9 & 127.6 & -0.7 & $(0.1)^{* *}$ & 127.5 \\
\hline Age (years) & 39.1 & 43.2 & -4.1 & $(0.1)^{* *}$ & 42.6 \\
\hline Share Male & 0.429 & 0.419 & +0.009 & $(0.003)^{* *}$ & 0.421 \\
\hline Chronic Illness & 0.576 & 0.609 & -0.034 & $(0.003)^{* *}$ & 0.604 \\
\hline Cancer & 0.069 & 0.100 & -0.030 & $(0.002)^{* *}$ & 0.095 \\
\hline Risk Score (HHS-HCC) & 1.016 & 1.041 & -0.025 & $(0.017)$ & 1.037 \\
\hline \multicolumn{6}{|l|}{ Medical Spending (\$/month) } \\
\hline Total spending & $\$ 310.0$ & $\$ 339.2$ & $-\$ 29.2$ & $(5.6)^{* *}$ & $\$ 334.7$ \\
\hline 6 months prior to switch & $\$ 325.9$ & $\mathrm{n} / \mathrm{a}$ & -- & & \\
\hline 6 months after switch & $\$ 298.7$ & $\mathrm{n} / \mathrm{a}$ & -- & & \\
\hline \multicolumn{6}{|l|}{ Duration and Switching } \\
\hline Duration enrolled: Prior to auto-switch & 11.9 & $\mathrm{n} / \mathrm{a}$ & -- & & \\
\hline After auto-switch & 9.9 & $\mathrm{n} / \mathrm{a}$ & -- & & \\
\hline Re-Switch Plans (w/in 3 mon.) & 0.153 & $\mathrm{n} / \mathrm{a}$ & -- & & \\
\hline
\end{tabular}

NOTE: The table shows average characteristics in the treatment group (100-150\% of poverty) for mid-year switchers (column 1) versus all other enrollees (column 2). Column (3) shows the difference between groups, and column (4) shows the average for the combined population of switchers and all others. Medical status variables (chronic illness and cancer) are based on diagnoses observed on claims for each year. Risk score (HHS-HCC) refers to the HHS Hierarchical Condition Category risk adjustment method used in the ACA Marketplaces. We impute this risk score for each enrollee at an annual level based on demographics and diagnoses observed on their claims. Medical costs refer to average medical spending (insurer-paid and cost sharing) per month enrolled; for switchers, we also show this separately for the (up to) 12 months enrolled prior to and after the mid-year switch. Share re-switch plans refers to the share of mid-year switchers who take the opportunity to switch plans again within three months. 